\title{
EL USO DEL ROBOT BEE-BOT COMO HERRAMIENTA DE APRENDIZAJE DE EMOCIONES EN LA ENSEÑANZA DEL ALUMNADO CON TRASTORNO DEL ESPECTRO AUTISTA
}

\author{
Elena Pérez Vázquez \\ elena.pv@ua.es \\ Gonzalo Lorenzo Lledó \\ glledo@ua.es \\ Asunción Lledó Carreres \\ asunción.lledo@ua.es \\ Alejandro Lorenzo Lledó \\ alejandro.Iorenzo@ua.es \\ Alba Gilabert Cerdá \\ agc136@alu.ua.es \\ Psicología evolutiva y didáctica \\ Universidad de Alicante
}

Fecha de Recepción: 18 Enero 2019

Fecha de Admisión: 30 Abril 2019

\section{RESUMEN}

Las Tecnologías de la Información y la comunicación (TIC) han experimentado en esta última década una gran expansión (Ferreyra, Méndez y Rodrigo, 2009). En este sentido, las TIC se han ido incorporando de forma progresiva en los centros escolares actuando como un recurso al servicio de una escuela inclusiva. Dicha escuela pretende dar respuesta a todo el alumnado del centro, haciendo especial hincapié en el alumnado con Necesidades Específicas de Apoyo Educativo (NEAE) (Arnaiz, 2013). Concretamente, entre dicho alumnado encontramos al alumnado con Trastorno del Espectro Autista (TEA). Siguiendo la $5^{\mathrm{a}}$ edición del Manual diagnóstico y estadístico de los trastornos mentales (American Psychiatric Association, 2013) el TEA es un trastorno del desarrollo caracterizado por déficits en dos áreas: (1) interacción social y comunicación y (2) patrones repetitivos de comportamientos, intereses y actividades. La amplia demanda de recursos por parte de los equipos docentes para lograr un proceso de enseñanza de calidad para el alumnado con TEA (Kim et al., 2018) y las características propias de las TIC que se alinean perfectamente con los principios metodológicos que deben guiar el proceso de enseñanza-aprendizaje de estos estudiantes, hacen que su uso experimente en crecimiento exponencial en este contexto. Entre el distinto tipo de TIC implementadas con este alumnado podemos encontrar la robótica (Dielh, Schmitt, Villano and Crowell, 2012). La utilización de esta tecnología ha aumentado en los últimos 20 años como consecuencia del aumento de investigaciones que avalan la robótica como una herramienta potencialmente efectiva para los niños con TEA (Dielh et al., 2018). En este sentido, los robots han permitido crear entornos de aprendizaje simples y controlados (Josman et al., 2018), disminuyendo la ansiedad que pueden llegar a provocarles. Sin embargo, aunque la mayoría de los estudios muestran efectos positivos (Goodrich et al., 2012; Huskens et al., 2013; Yun, Choi y Park, 2015), la mayoría de estos estudios presentan limitaciones metodológicas y poseen carácter exploratorio debido al bajo número de participantes involucrados. Asimismo, el uso de robots es limitado por los grandes costes que 


\section{EL USO DEL ROBOT BEE-BOT COMO HERRAMIENTA DE APRENDIZAJE DE EMOCIONES EN LA ENSEÑANZA DEL ALUMNADO CON TRASTORNO DEL ESPECTRO AUTISTA}

deben ser asumidos por parte de los centros educativos y por la falta de disponibilidad d estos dispositivos (Alexander et al., 2011). En este sentido, el uso de robots en contextos educativos resulta escaso. De este modo, el propósito del estudio ha sido realizar una intervención para la comprensión, reconocimiento y expresión de emociones con la utilización del robot Bee-Bot, un robot asequible económicamente para los centros educativos. A través de una metodología mixta mediante estudio de caso, han participado 10 alumnos/as de edades comprendidas entre 5 y 18 años, pertenecientes a un Unidad Específica de Comunicación y Lenguaje (UECYL). Esta aula pertenece a un colegio de Educación Infantil y Primaria de Alicante. El instrumento utilizado ha sido un pretest-postest con diferentes dimensiones. Los resultados de la presente investigación señalan que los alumnos presentan una evolución bastante notable en el aprendizaje de las emociones básicas, así como en aspectos relacionados con la programación y la orientación espacial. A modo de conclusión, podemos afirmar que, la robótica constituye una herramienta de fuerte impacto en la intervención socioemocional del alumnado con TEA, creando escenarios sencillos donde aprender y practicar distintas habilidades. Este trabajo se enmarca dentro de la línea de investigación del grupo de investigación de la Universidad de Alicante IncluTic (VIRGROB-321).

Palabras clave: robótica; Bee-Bot; gestión emocional; trastorno del espectro autista

\section{ABSTRACT}

The use of the bee-bot robot as a learning tool for emotions in the teaching of students with Autism Spectrum Disorder. The Information and Communication Technologies (ICT) have experimented in this last decade a great expansion (Ferreyra, Méndez and Rodrigo, 2009). In this sense, ICTs have been progressively incorporated in schools acting as a resource at the service of an inclusive school. This school aims to respond to all students in the center, with special emphasis on students with Specific Educational Support Needs (Arnaiz, 2013). Specifically, among these students we find the student body with Autism Spectrum Disorder (ASD). Following the 5th edition of the Diagnostic and Statistical Manual of Mental Disorders (American Psychiatric Association, 2013), TEA is a developmental disorder characterized by deficits in two areas: (1) social interaction and communication and (2) repetitive patterns of behaviors, interests, and activities. The ample demand for resources on the part of teaching teams to achieve a quality teaching process for students with TEA (Kim et al., 2018) and the characteristics of ICT that are perfectly aligned with the methodological principles that should guide the teaching-learning process of these students, make their use experience exponential growth in this context. Among the different types of ICT implemented with these students we can find robotics (Dielh, Schmitt, Villano and Crowell, 2012). The use of this technology has increased in the last 20 years as a consequence of the increase in research that supports robotics as a potentially effective tool for children with ASD (Dielh et al., 2018). In this sense, robots have permitted the creation of simple and controlled learning environments (Josman et al., 2018), reducing the anxiety that can cause them. However, although most studies show positive effects (Goodrich et al., 2012; Huskens et al., 2013; Yun, Choi and Park, 2015), most of these studies have methodological limitations and have exploratory character due to the low number of participants involved. Likewise, the use of robots is limited by the large costs that must be assumed by schools and by the lack of availability of these devices (Alexander et al., 2011). In this sense, the use of robots in educational contexts is limited. Thus, the purpose of the study has been to perform an intervention for the understanding, recognition and expression of emotions with the use of the BeeBot robot, an affordable robot for schools. Through a mixed methodology by means of a case study, 10 students between the ages of 5 and 18, belonging to a Specific Communication and Language Unit (UECYL), have participated. This classroom belongs to a school of Infant and Primary Education 
of Alicante. The instrument used has been a pretest-postest with different dimensions. The results of the present research indicate that the students present a quite remarkable evolution in the learning of basic emotions, as well as in aspects related to programming and spatial orientation. To conclude, we can say that robotics is a tool with a strong impact on the socio-emotional intervention of students with TEA, creating simple scenarios in which to learn and practice different skills. This work is part of the research line of the research group of the University of Alicante IncluTic (VIRGROB321).

Keywords: robotics; Bee-Bot, emotional management; autism spectrum disorder

\section{INTRODUCTION}

Sin duda el aumento significativo en la prevalencia de casos diagnosticados como trastornos del espectro autista en las últimas décadas viene determinado por un mayor conocimiento del trastorno, a la disponibilidad de mejores herramientas de diagnóstico, así como a los cambios introducidos en los manuales de diagnóstico (Blumberg, Bramlett, Kogan, Schieve, Jones \& Lu, 2013; Schieve, 2011; Tárraga- Mínguez \& Sanz-Cervera, 2018).

Actualmente, se conoce a los Trastornos del Espectro Autista (TEA) como un trastorno del desarrollo caracterizado por deficiencias en dos áreas fundamentales: (1) interacción social y de comunicación y (2) patrones repetitivos de comportamientos, intereses o actividades (American Psychiatric Association, 2013). El rasgo distintivo del alumnado con trastornos del Espectro autista son las dificultades para comprender las conductas sociales propias y de las personas de su entorno.

A pesar de no existir un consenso en cuanto a la etiología del Trastorno del Espectro Autista, sí que han sido constatadas un tipo de intervenciones para mejorar la calidad de vida de las personas con TEA (Tárraga-Mínguez y Sanz-Cervera, 2018). En este sentido se ha mostrado la efectividad de intervenciones basadas en el Análisis Conductual Aplicado (Makryianni, Gena, Katoudi \& Galanis, 2018), en la metodología TEACCH (Sanz-Cervera, Fernández-Andrés, Pastor-Cerezuela \& TárragaMínguez, 2018), en historias sociales (McGill, Baker \& Busse, 2014), en terapia de juego basado en el niño (Hillman, 2018) y en nuevas tecnologías (Pinel, Aguiló \& Adrover-Roig

Más concretamente, el uso de las tecnologías de la información y la comunicación (TIC) ha aumentado considerablemente en los últimos años, llegando al mundo de la educación (Ke \& Im, 2013). Autores como Tortosa (2004) afirman que las TIC se alinean perfectamente con los principios metodológicos que deben guiar el proceso de enseñanza-aprendizaje de estos estudiantes y pueden ser determinantes para mejorar la calidad de vida de las personas con discapacidad y para propiciar el acceso al curriculum oficial, posibilitando así una inclusión completa.

Entre dichas tecnologías encontramos a la robótica. Actualmente, el uso de la robótica destinada a mejorar la gestión emocional de los alumnos con TEA ha aumentado de forma considerable (Michaud, Salter, Duquette \& Laplante, 2007; Salter, Michaud \& Larouche, 2010). Específicamente, la robótica permite aumentar el nivel de motivación y participación del alumnado en el proceso de enseñanza-aprendizaje (Scasellati, Admoni \& Mataric, 2012, American Psychiatric Association, 2013). Por otro lado, permite crear entornos más simples y controlados que reducen la posibilidad de error (Goodrich et al., 2012, Kozima, Michalowski \& Nakagawa, 2009). Sin embargo, al ser un campo reciente cuenta con un gran número de estudios de carácter exploratorio que no permiten generalizar los resultados (Diehl et al., 2012)

Esta investigación forma parte de una investigación más amplia iniciada durante el curso 20172018 en la ciudad de Alicante, cuyo objetivo es comprobar si el uso del robot Bee-bot es una herramienta tecnológica que ayuda a las personas con TEA en la gestión de las emociones. Así pues, esta 


\section{EL USO DEL ROBOT BEE-BOT COMO HERRAMIENTA DE APRENDIZAJE DE EMOCIONES EN LA ENSEÑANZA DEL ALUMNADO CON TRASTORNO DEL ESPECTRO AUTISTA}

investigación en concreto pretende realizar una aproximación científica relativa al uso del robot para trabajar una emoción básica y comparar las percepciones iniciales de los profesores sobre el manejo emocional de dos estudiantes con TEA con las percepciones de los investigadores tras la aplicación de esta tecnología. En este sentido, para lograr este objetivo principal, hemos establecido las siguientes preguntas de investigación:

¿Coinciden las percepciones del profesor con las percepciones del investigador con respecto a la capacidad de identificación de emociones de los estudiantes con TEA?

¿Mejora la capacidad de identificar las emociones del estudiante con TEA tras la utilización del robot Bee-Bot?

$¿$ Coinciden las percepciones del profesor con las percepciones del investigador con respecto a la capacidad de comprensión emocional de los estudiantes con TEA?

¿Mejora la capacidad de comprensión emocional del estudiante con TEA tras la utilización del robot Bee-Bot?

¿Coinciden las percepciones del maestro con las percepciones del investigador con respecto a la capacidad de los estudiantes para expresar emociones con TEA?

¿Mejora la capacidad de expresión emocional del estudiante con TEA tras la utilización del robot Bee-Bot?

\section{METODOLOGÍA}

Se ha llevado a cabo un estudio basado en una metodología cuantitativa descriptiva.

\section{Contexto y participantes}

El presente estudio de carácter exploratorio forma parte de uno de mayor envergadura contextualizado en la provincia de Alicante. Concretamente, por lo que respecta a la presente investigación, Ios participantes pertenecen a un aula de comunicación y lenguaje (CYL) ubicada en un centro ordinario concertado. El aula está conformada por 10 alumnos/as de edades comprendidas entre 5 y 18 años de los cuales seis han sido seleccionados para el presente estudio.

La tabla 1 refleja las características de los participantes del estudio. Todos ellos presentan un diagnóstico de Trastorno del Espectro Autista. Específicamente, siguiendo sus informes pedagógicos basados en el Manual Diagnóstico y Estadístico de los Trastornos Mentales (DSM-5), los niveles de TEA del alumnado están comprendidos entre el 1 y el 2, siendo más mayoritario este último. Por otro lado, las edades del alumnado están comprendidas entre 6 y 16 años. Es importante señalar que todos ellos utilizan una comunicación verbal y presentan un nivel actual de competencia inferior al de su curso de referencia.

Table 1. Características de los participantes

\begin{tabular}{ccccccc}
\hline & Estudiante & Estudiante & Estudiante & Estudiante & Estudiante & Estudiante \\
& 1 & 2 & 3 & 4 & 5 & 6 \\
\hline Edad & 6 & 10 & 6 & 12 & 16 & 14 \\
Nivel de TEA & 2 & 2 & 2 & 1 & 1 & 2 \\
$\begin{array}{c}\text { Comunicación } \\
\text { Nivel actual } \\
\text { de }\end{array}$ & 5 años & $4^{\circ} \mathrm{EP}$ & $1^{\circ} \mathrm{EP}$ & $4^{\circ} \mathrm{EP}$ & $1^{\circ} \mathrm{ESO}$ & $1^{\circ} \mathrm{ESO}$ \\
$\begin{array}{c}\text { competencia } \\
\text { combal }\end{array}$ & & & & & & \\
\hline
\end{tabular}




\section{Instrumento}

La recogida de datos precisa de la utilización de varios instrumentos. En primer lugar, para la confirmación del diagnóstico de alumno con Trastorno del Espectro Autista se utilizó la Escala de Evaluación de Gilliam para diagnóstico de autismo (GARS-2).

Por otro lado, se diseñaron dos instrumentos para llevar a cabo la recopilación de información. En primer lugar, se diseñó e implementó un cuestionario con el objetivo de conocer la percepción de los profesores sobre la gestión emocional de sus alumnos. Dicho cuestionario actúa como pretest del proceso en su conjunto. Asimismo, dicho cuestionario se divide en tres dimensiones: (1) identificación de las emociones, (2) comprensión emocional y (3) expresión de las emociones. Todas las dimensiones contienen diferentes ítems, los cuales se miden bajo una escala de Likert.

En segundo lugar, se llevó a cabo el diseño de un segundo cuestionario basado en estas tres dimensiones, el cual permitía el seguimiento de los resultados de las sesiones. Este segundo cuestionario (amplía las tres dimensiones al incluir un mayor número de ítems en cada una de ellas.

Ambos cuestionarios se encuentran interrelacionados como se puede observar en la tabla 2.

Tabla 2. Interrelación de los ítems entre los instrumentos.

\begin{tabular}{ll}
\hline $\begin{array}{l}\text { Dimensiones del cuestionario sobre la } \\
\text { percepción de los profesores (pretest) }\end{array}$ & $\begin{array}{l}\text { Dimensiones del cuestionario destinado al } \\
\text { registro de las sesiones. }\end{array}$ \\
\hline Identificación de emociones & Asociación color-emoción \\
& $\begin{array}{l}\text { Identificación de la expresión facial de la } \\
\text { emoción en un dibujo }\end{array}$ \\
& $\begin{array}{l}\text { Identificación de la expresión facial de la } \\
\text { emoción en un emoticón }\end{array}$ \\
& Identificación de la expresión facial de la \\
& emoción en una fotografía.
\end{tabular}

Comprensión emocional

Comprender las acciones y comportamientos de las emociones.

Reconocimiento de la emoción a través de la expresión corporal de otras personas.

Expresión de emociones Expresión corporal de las emociones básicas.

\section{Proceso}

El estudio se realizó durante nueve sesiones. La primera sesión tuvo como objetivo de buscar la familiarización del alumnado con los comandos del robot, así como a los investigadores. Por otra parte, durante el resto de las sesiones las actividades se realizaron de forma progresiva y en periodos cortos de tiempo, debido a las características de los alumnos. De esta manera, la duración de las sesiones rondaba entre los 15 y los 25 minutos por alumno/a. Por último, las dos últimas sesiones (sesión 8 y sesión 9) se realizaron de forma completa evaluando todos los ítems en la misma sesión.

Las sesiones se llevaron a cabo individualmente durante los meses de febrero y marzo del presente curso escolar, 2018-2019. El encargado principal de guiar al alumno en el proceso fue el investigador. Sin embargo, bien es cierto que los tutores estuvieron presentes en todas las sesiones. 


\section{EL USO DEL ROBOT BEE-BOT COMO HERRAMIENTA DE APRENDIZAJE DE EMOCIONES EN LA ENSEÑANZA DEL ALUMNADO CON TRASTORNO DEL ESPECTRO AUTISTA}

\section{RESULTS}

A continuación, se exponen los principales resultados del estudio. Concretamente, se presentan Ios resultados atendiendo a las tres dimensiones de los cuestionarios: (Figura 1) Identificación de emociones, (Figura 2) Comprensión emocional y (Figura 3) Expresión de emociones.

Figura 3. Dimensión 3: Expresión de emociones
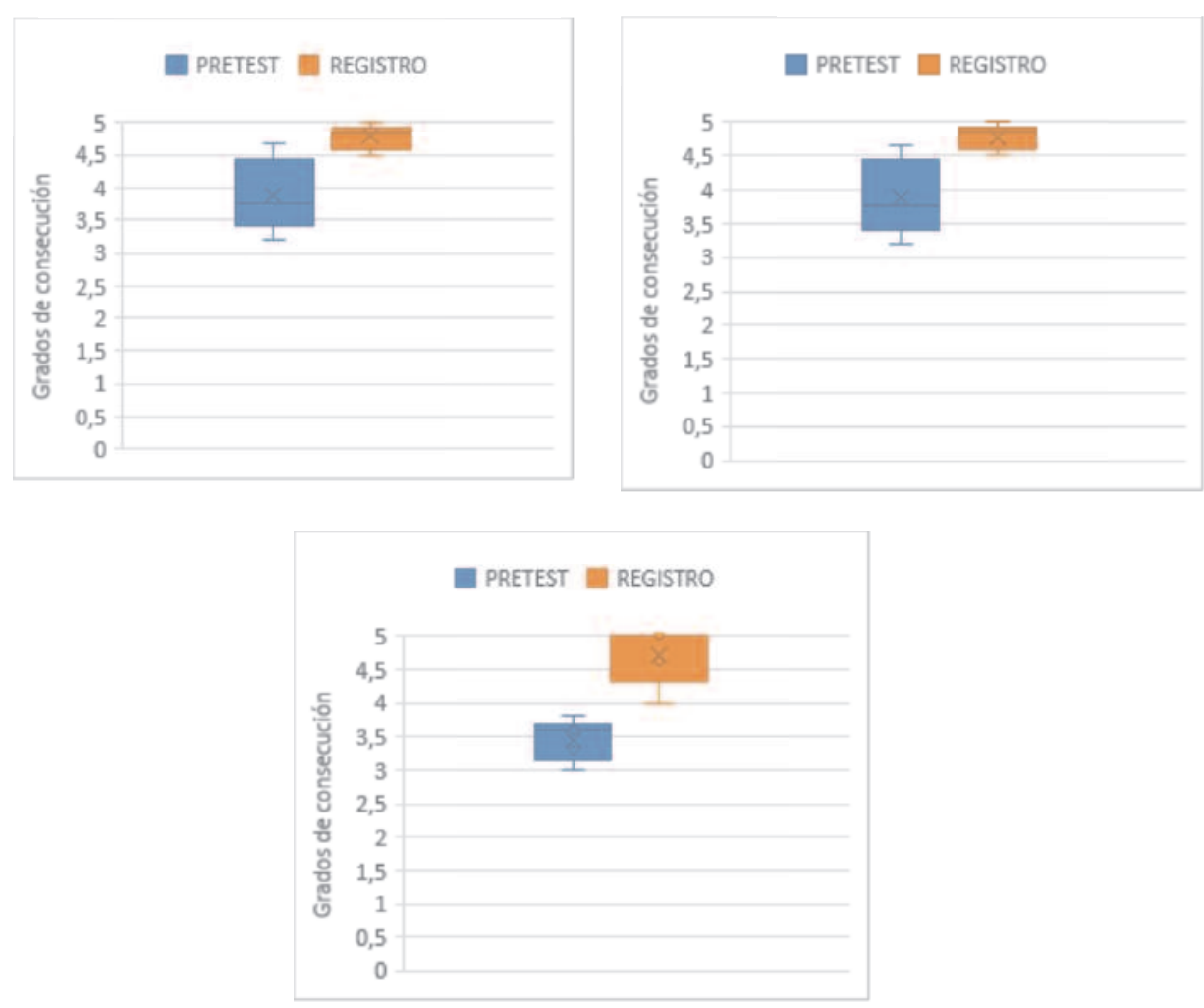

La distribución de valores en los grados de consecución referentes al pretest es simétrica, no presentando valores atípicos en ninguna de ellas. Sin embargo, por lo que respecta a los grados de consecución relativos al registro relativos a las dimensiones de (1) identificación de emociones y (2) comprensión emocional se observa una distribución asimétrica mínima. Por otro lado, la asimetría de los grados de consecución relativos a la dimensión (3) expresión de emociones es bastante mayor a la de las dos dimensiones anteriores.

Por lo que respecta a la mediana (y la media), como se puede observar en las figuras, para todas las dimensiones aumenta. Asimismo, se observan diferencias entre las dispersiones, siendo menor la dispersión de los datos relativos a los grados de consecución del registro de sesiones.

Por último, como se puede observar existe una notable diferencia entre los datos recogidos al principio de las sesiones mediante el pretest, el cual fue completado por el profesorado, y los datos en las últimas sesiones. Más concretamente, existe una mejora de los resultados, alcanzando una puntuación muy elevada en las tres dimensiones estudiadas. 


\section{CONCLUSIONES}

Los resultados de nuestra investigación nos permiten sacar las siguientes conclusiones.

En primer lugar, las percepciones del profesorado del alumnado con TEA difieren de las percepciones del investigador con respecto a la capacidad de identificación de emociones de estos estudiantes, así como en la capacidad de comprensión emocional y en la expresión de emociones, siendo las percepciones de los investigadores más positivas. En esta misma línea y enlazando con el resto de las preguntas de investigación, podemos señalar que se percibe una notable mejora de la capacidad del alumnado con TEA para gestionar sus emociones tras la utilización del robot como herramienta de aprendizaje. Sin embargo, es pronto para señalar si las diferencias existentes son significativas.

De esta manera, y ante la necesidad de confirmar estos resultados, se propone la celebración de varias sesiones en las que se trabajen todas las dimensiones, para poder confirmar la literatura actual. Además, el estudio se extiende al trabajo de otras emociones básicas como la tristeza, la ira y el miedo. Por otro lado, la muestra de participantes se ampliará a un máximo de veinticinco estudiantes con un diagnóstico de trastorno del espectro autista (TEA).

\section{AGRADECIMIENTOS}

Este trabajo ha sido realizado por miembros del grupo de investigación IncluTIC de la Universidad de Alicante. Los autores agradecen sinceramente a la Escuela Agustinos Alicante por su apoyo en esta investigación y por su compromiso en la enseñanza de estudiantes con trastornos del espectro autista.

\section{REFERENCES}

American Psychiatric Association. (2013) Diagnostic and statistical manual of mental disorders, 5th edn. Arington, VA: American Psychiatric Publishing.

Aresti-Bartolome, N., \& Garcia-Zapirain, B. Technologies as support tools for persons with autistic spectrum disorder: A systematic review, Int. J. Environ. Res. Public Health, 11, 7767-7802, 2014.

Arnaiz, P. (2013). Educación Inclusiva: una escuela para todos. Ed. Aljibe

Blumberg, S.J., Bramlett, M.D., Kogan, M.D., Schieve, L.A. Jones, J.R. \& Lu, M.C. (2013). Changes in prevalence of parent-reported autism spectrum disorder in school-aged U.S. children: 2007 to 2011-2012. National Health Statistics Reports, 65(20), 1-12.

Diehl, J.J., Schmitt, L.M., Villano, M., \& Crowell, C.R. (2012). The clinical use of robots for individuals with Autism Spectrum Disorders: A critical review. Research in autism spectrum disorders, 6(1), 249-262.

Ferreyra, J.A., Méndez, A., \& Rodrigo, M.A. (2009) El uso de las TIC en la Educación Especial: Descripción de un sistema informático para niños discapacitados visuales en Etapa Preescolar. Revista Iberoamericana de Tecnología en Educación y Educación en Tecnología, 3, 55-62.

Goodrich, M.A., Colton, M., Brinton, B., Fujiki, M., Alan Atherton, J., Robinson, L., Acerson, A. (2012). Incorporating a robot into an autism therapy team. IEEE Intelligent Systems, 27(2), 52. http://dx.doi.org/10.1109/MIS.2012.40

Hillman, H. (2018). Child-Centered Play Therapy as an Intervention for Children With Autism: A Literature Review. International Journal of Play Therapy, vol. 27(4), 198-204.

Huskens, B., Verschuur, R., Gillesen, J., Didden, R., \& Barakova, E. (2013). Promoting questionasking in school-aged children with autism spectrum disorders: Effectiveness of a robot intervention compared to a human-trainer intervention. Developmental neurorehabilitation, 16(5), 345-356. 


\section{EL USO DEL ROBOT BEE-BOT COMO HERRAMIENTA DE APRENDIZAJE DE EMOCIONES EN LA ENSEÑANZA DEL ALUMNADO CON TRASTORNO DEL ESPECTRO AUTISTA}

Josman, N., Ben-Chaim, H., Friedrich, H., \& Weiss P.(2008). Effectiveness of virtual reality for teaching street-crossing skills to children and adolescents with autism. Int J Disabil Hum Dev, 7, 49-56.

Ke, F., \& Im, T. (2013).Virtual-reality-based social interaction training for children with highfunctioning autism. J. Educ. Res., 106(6), 441-46.

Kim, E.S., Berkovits, L.D., Bernier, E.P., Leyzberg, D., Shic, F., Paul, R. \& Scassellati, B. (2013). Social robots as embedded reinforces of social behavior in children with autism. Journal of Autism and Developmental Disorders, 43, 1038-1049.

Kozima, H., Michalowski, M.P., \& Nakagawa, C. (2009). A playful robot for research therapy and entertainment. International Journal of Social Robotics, 1(1), 3-18.

Makrygianni, M. K., Gena, A., Katoudi, S., y Galanis, P. (2018). The effectiveness of applied behavior analytic interventions for children with Autism Spectrum Disorder: A meta-analytic study. Research in Autism Spectrum Disorders, 51, 18-31.

McGill, RJ., Baker, D., \& Busse, R. T. (2015). Social Story ${ }^{\top M}$ interventions for decreasing challenging behaviours: a singlecase meta-analysis 1995-2012. Educational Psychology in Practice, 31(1), 21-42.

Michaud, F., Salter, T., Duquette A., \& Laplante, J.F. (2007). Perspectives on mobile robots used as tools for pediatric rehabilitation. Assistive Technology, 19, pp.14-29.

Pinel, V., Rendon, L.A ., and Adrover-Roig, D. (2018). Los robots sociales como promotores de la comunicación en los Trastornos del Espectro Autista (TEA). Let. Hoje, 53(1), 39-47. http://dx.doi.org/10.15448/1984-7726.2018.1.28920

Salter, T., Michaud F., \& Larouche, H. (2010). How wild is wild? A taxonomy to categorize the wildness of child-robot interaction. International Journal of Social Robotics, 2(4), pp.405-415.

Sanz-Cervera, P., Fernández-Andrés, M. I., Pastor-Cerezuela, G., y Tárraga-Mínguez, R. (2018). Efectividad de las intervenciones basadas en metodología TEACCH en el trastorno del espectro autista: un estudio de revisión. Papeles del Psicólogo, 39(1), 40-50.

Scasellati, B., Admoni, H., \& Mataric, M. (2012). Robots for use in autism research. Annu. Rev.Biomed.Eng, 14, 275-294.

Schieve, L.A., Rice, C., Devine, O., Maenner, M.J. Lee, L.C., Fitzgerald, R., Wingate, M.S., D. Schendel, S. Pettygrove, K. van Naarden Braun. (2011). Have secular changes in perinatal risk factors contributed to the recent autism prevalence increase? Development and application of a mathematical assessment model. Annals of Epidemiology, 21(12),930-945.

Tárraga- Mínguez R., \& Sanz-Cervera, P. (2018). ¿Qué estrategias de intervención funcionan en la educación de los niños con trastorno del espectro autista? Revisión de evidencias en la literatura científica. Reidocrea, 7(22), 279-287. 